ISSN electrónico: 2602-8069

\title{
MICROSCOPIO ELECTRÓNICO DE BARRIDO Y ELUCUBRACIONES HISTÓRICAS. EL ARTESONADO MUDÉJAR DEL SANTUARIO DE TACORONTE (TENERIFE).
}

Scanning electron microscope and historical reflections. The mudéjar coffered ceiling of the Tacoronte's sanctuary (Tenerife).

Antonio Marrero Alberto Universidad Adolfo Ibáñez (Chile) antoniomarreroalberto@hotmail.es 


\title{
Antonio Marrero Alberto \\ Microscopio electrónico de barrido y elucubraciones históricas. El artesonado mudéjar del santuario de Tacoronte (Tenerife)
}

\begin{abstract}
RESUMEN
En los trabajos de restauración de obras de arte, se hace necesaria la realización de determinados análisis, para la identificación de los materiales constituyentes de dichas piezas. El objetivo de este artículo es poner en valor la importancia de las técnicas analíticas y la interpretación de los resultados que se deriven de las mismas, para posibles elucubraciones y teorizaciones por parte de los historiadores del arte, generando reflexión en torno a la motivación del porqué del empleo de esos materiales. Se ha tomado como ejemplo el Artesonado de la Capilla Mayor del Santuario del Cristo de Tacoronte (Tenerife) y las muestras estratigráficas obtenidas del mismo, atendiendo a los seis colores usados para el policromado de dicha obra. Se han empleado microscopios ópticos y electrónicos de barrido, y, tras identificar los pigmentos utilizados en la decoración de la cubierta, postulamos una teoría que, más allá de la evidente función decorativa, ahonde en la idea de un uso animista de la techumbre, donde se ponen al servicio de la devocionada imagen del Varón de Dolores, todos los elementos que identifican y enriquecen al pueblo de Tacoronte (agrícolas, ganaderos, pesqueros, e inclusos antropológicos), para su protección.
\end{abstract}

Palabras clave: Microscopio electrónico de barrido, historia del arte, artesonado, Tacoronte.

\section{ABSTRACT}

In the restoration of works of art, it is necessary to carry out certain analyzes, for the identification of the constituent materials of these pieces. The objective of this article is to value the importance of analytical techniques and the interpretation of the results that derive from them, for possible elucubrations and theorisations by art historians, generating reflection on the motivation of the use of those materials. The Coffered ceiling of the Main Chapel of the Christ church from Tacoronte (Tenerife) and the stratigraphic samples obtained from it have been taken as an example, taking into account the six colors used for the polychrome of this work. Optical and scanning electron microscopes have been used, and, after identifying the pigments used in the decoration of the roof, we postulate a theory that, beyond the obvious decorative function, goes deeper into the idea of an animistic use of the roof, where they are put at the service of the devoured image of the Varón de Dolores, all the elements that identify and enrich the people of Tacoronte (agricultural, livestock, fishing, and even anthropological), for its protection.

Key words: Scanning electron microscope, art history, coffered ceiling, Tacoronte. 


\section{Antonio Marrero Alberto \\ Microscopio electrónico de barrido y elucubraciones históricas. El artesonado mudéjar del santuario de Tacoronte (Tenerife)}

\section{INTRODUCCIÓN}

El concepto de multidisciplinariedad se impone en todos los ámbitos y, en el caso de las obras de arte, adquiere una posición preeminente, demostrando que para la ejecución de un trabajo completo y efectivo, se deben atender todas las disciplinas que en él puedan tener cabida. Historiadores del arte, arquitectos, carpinteros, químicos y, no menos importantes, expertos en técnicas de análisis, forman un grupo heterogéneo donde cada uno aporta su conocimiento, estableciendo así un protocolo de actuación que se vislumbra como el más idóneo de todos los existentes.

De entre todo ellos, los encargados de la realización e interpretación de los ya mencionados análisis, se erigen como apoyo fundamental en la investigación e intervención restaurativa sobre un bien patrimonial. Sus resultados son fuentes de conocimiento para historiadores del arte, dando veracidad y solidez a los postulados que plantean.

En el caso que es objeto de esta investigación, destaca el análisis al que se han sometido una serie de muestras tomadas del artesonado mudéjar de la capilla mayor del Santuario del Santísimo Cristo de los Dolores (Tacoronte), siendo la Microscopía Electrónica de Barrido con Espectrometría de rayos-X,${ }^{1}$ detector de productos inorgánicos (pigmentos y cargas), y los resultados obtenidos con sus respectivas interpretaciones, la base para establecer elucubraciones y planteamientos desde una perspectiva históricoartística.

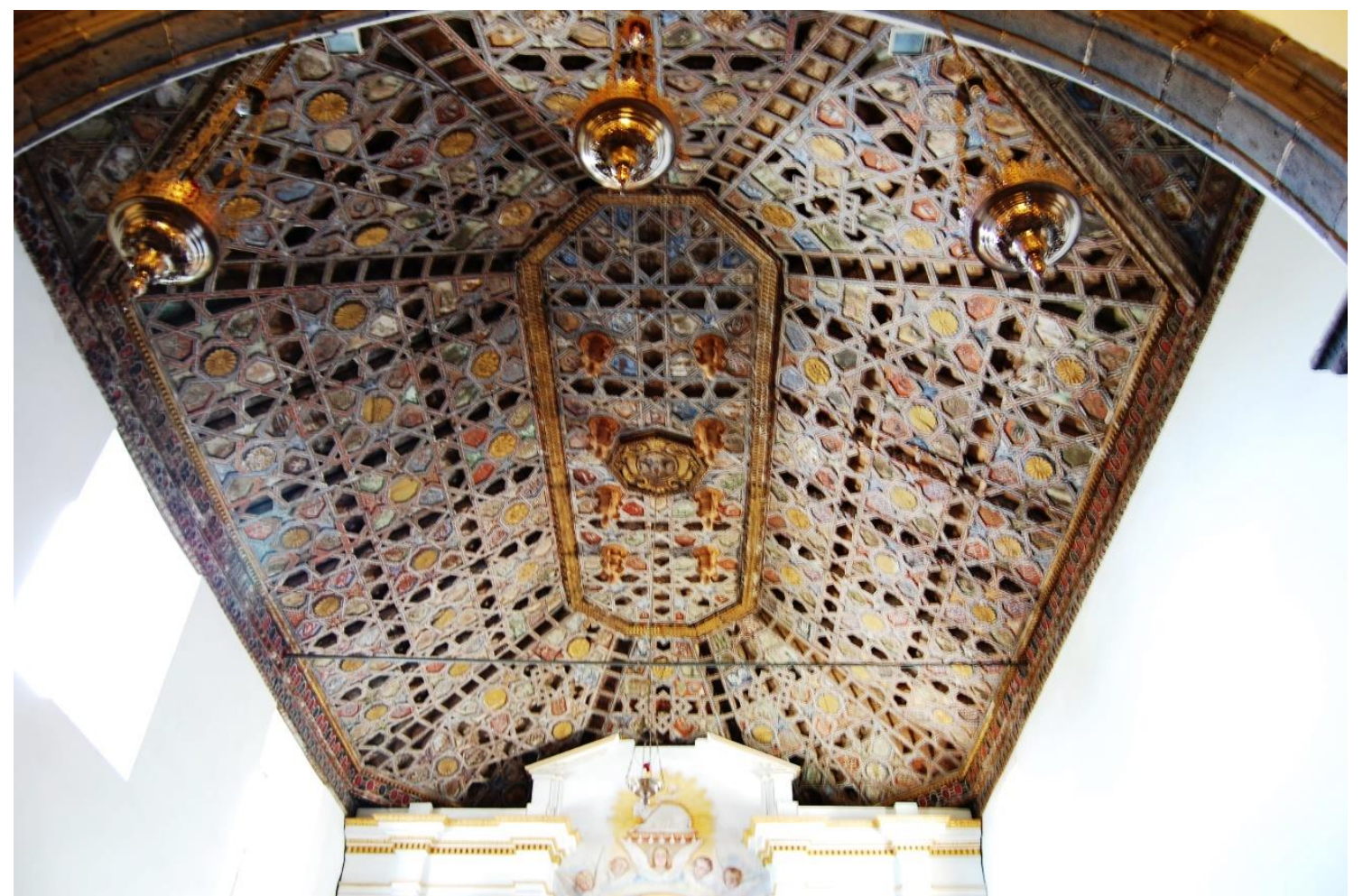

Figura 1. Artesonado mudéjar, Capilla Mayor del Santuario del Santísimo Cristo de los Dolores, Tacoronte (Tenerife, Islas Canarias, España).

${ }^{1}$ Scanning Electron Microscopy - Energy Dispersive X-ray spectroscopy, en adelante, SEM/EDX. 


\section{Antonio Marrero Alberto \\ Microscopio electrónico de barrido y elucubraciones históricas. El artesonado mudéjar del santuario de Tacoronte (Tenerife)}

Partiendo de los estudios histórico-artísticos que se han escrito sobre el edificio, ${ }^{2}$ se sabe que el Santuario nació a partir de la ermita de San Sebastián, de cuya existencia contamos con fuentes primarias desde 1599 y que se situó en los límites del municipio. Ampliada a lo largo del siglo XVII, hasta obtener las dimensiones con las que cuenta hoy en día, formó parte de un conjunto conventual agustino patrocinado por el Capitán

D. Tomás Pereira de Castro y Ayala, hasta la desamortización de 1837, la cual autorizaba al gobierno para destinar a establecimientos de utilidad pública los conventos suprimidos, aunque ya por esas fechas la comunidad estaba prácticamente extinta, convirtiéndose su morada en Casa Consistorial, mientras que la iglesia pasó a depender de la parroquia de Santa Catalina de Alejandría Mártir, también en Tacoronte.

La armadura de la capilla mayor ${ }^{3}$-que es de la que trata este estudio- es un artesonado de técnica ataujerada, ejecutado en 1664 y policromado en 1665. Las maderas ensambladas en fina labor de lacería forman octógonos, crucetas, estrellas, etc. Una combinación de vivos colores se reparte por toda la techumbre, de cuyo almizate cuelgan piñas de mocárabes. Por sus características formales y desarrollo iconográfico, resulta uno de los artesonados más ricos y elaborados de Canarias. Sus dimensiones son considerables, con un ancho de 7,824 m, una profundidad de 12,196m y una altura mínima de 7,347 m en la cinta y máxima de 9,358 $\mathrm{m}$ en el almizate. La capilla data de los mismos años que el resto del edificio, salvo la policromía del artesonado, que tras el descubrimiento de una fecha en uno de los cuartillejos del almizate y analizada por acreditados paleógrafos del Departamento de Paleografía de la Universidad Complutense de Madrid, podemos afirmar que data de $1665^{4}$.

Según la estructura y composición, se observa una techumbre rectangular de ocho vertientes. Presenta almizate con ocho perillones de una intervención del siglo XX, plafón central y cinta perimetral. De lima moamar, se repiten los lazos de ocho que invaden todo el artesonado, generándose, a su vez, cuadrados que se centran con soles y flores doradas, y que conforman tres grandes bandas. La lacería está encolada y clavada, salvo la que comunica los lazos por encima de las limas y las que dividen los cuadrados, que se sujetan por los extremos. Este esquema que mezcla dichos lazos con cuadrados, y distribuye la lacería por toda la superficie, se copiará en otras techumbres existentes en las islas ${ }^{5}$. El paso del ochavo al cuadrado se realiza mediante una cinta que salva el desnivel y una

2 Casas Otero, J. (1987). Estudio Histórico Artístico de Tacoronte. Santa Cruz de Tenerife, España: Aula de Cultura de Tenerife, Excmo. Cabildo Insular de Tenerife, p. 132. Fraga González, M. C. (1977). La arquitectura mudéjar en Canarias. Santa Cruz de Tenerife, España: Aula de Cultura de Tenerife, pp. 99-100. Acosta Trujillo, R. (2005). Las techumbres mudéjares policromadas en Canarias. Estudio de tres casos. En VIII Simposio sobre centros históricos y patrimonio cultural de Canarias (pp. 75-82). San Cristóbal de La Laguna, España: Fundación CICOP. Bonnet Suárez, S. F. (Julio-Agosto-Septiembre 1944). Tacoronte y sus templos. El Museo Canario, V (11), p. 36. Bonnet, S.F. (Noviembre 1961). El convento y la iglesia de San Agustín, en Tacoronte. La Tarde. Marrero Alberto (2017) ¿Mudéjar o Mudejárico? Compendio historiográfico y reflexión crítica. El uso del término mudéjar en canarias. Revista de Historia Canaria (199), pp. 105-140.

\footnotetext{
${ }^{3}$ Ampliamente estudiada en: Marrero Alberto, A. (2016). Los artesonados mudéjares policromados en la isla de Tenerife (Tesis doctoral). Universidad de La Laguna, San Cristóbal de La Laguna, España.

${ }^{4}$ Marrero Alberto, A. (2013). Los Artesonados Mudéjares Policromados en la isla de Tenerife. Aspectos técnicos y su conservación y restauración. En Trabajos de Fin de Máster: Máster Universitario en Conservación y restauración de Bienes Culturales 2006/2010. Trabajos seleccionados (pp. 1-65). Valencia, España: Edición digital Diazotec, Universidad Politécnica de Valencia.

${ }^{5}$ Marrero Alberto, A. (Julio - diciembre 2018). Armaduras: planos póstumos y reconstrucción. La Iglesia de la Concepción (Los Realejos). Revista Estoa (13).
} 


\section{Antonio Marrero Alberto \\ Microscopio electrónico de barrido y elucubraciones históricas. El artesonado mudéjar del santuario de Tacoronte (Tenerife)}

contrahuella con una sucesión de óvalos. Los cuadrantes son planos con lacería encolada y clavada, y perillones iguales a los del almizate. La solera se conforma con dos frisos de óvalos que dan paso al plano vertical.

En cuanto al estudio iconográfico, las limas y alfardas ataujeradas presentan decoración blanca, directa sobre la madera, con numerosos tipos de representación (hojarasca, estrellas, abstracción, etc.). El fondo de las formas que genera el trabajo de lacería, es de una riqueza polícroma sin igual y con representaciones de todo tipo: frutas, hortalizas, animales, utensilios de caza, cocina, pesca, ángeles, faunos, formas geométricas, etc. La cinta del almizate y la solera mezcla dorado y corlas. En el pinjante del almizate se representa al Espíritu Santo ${ }^{6}$.

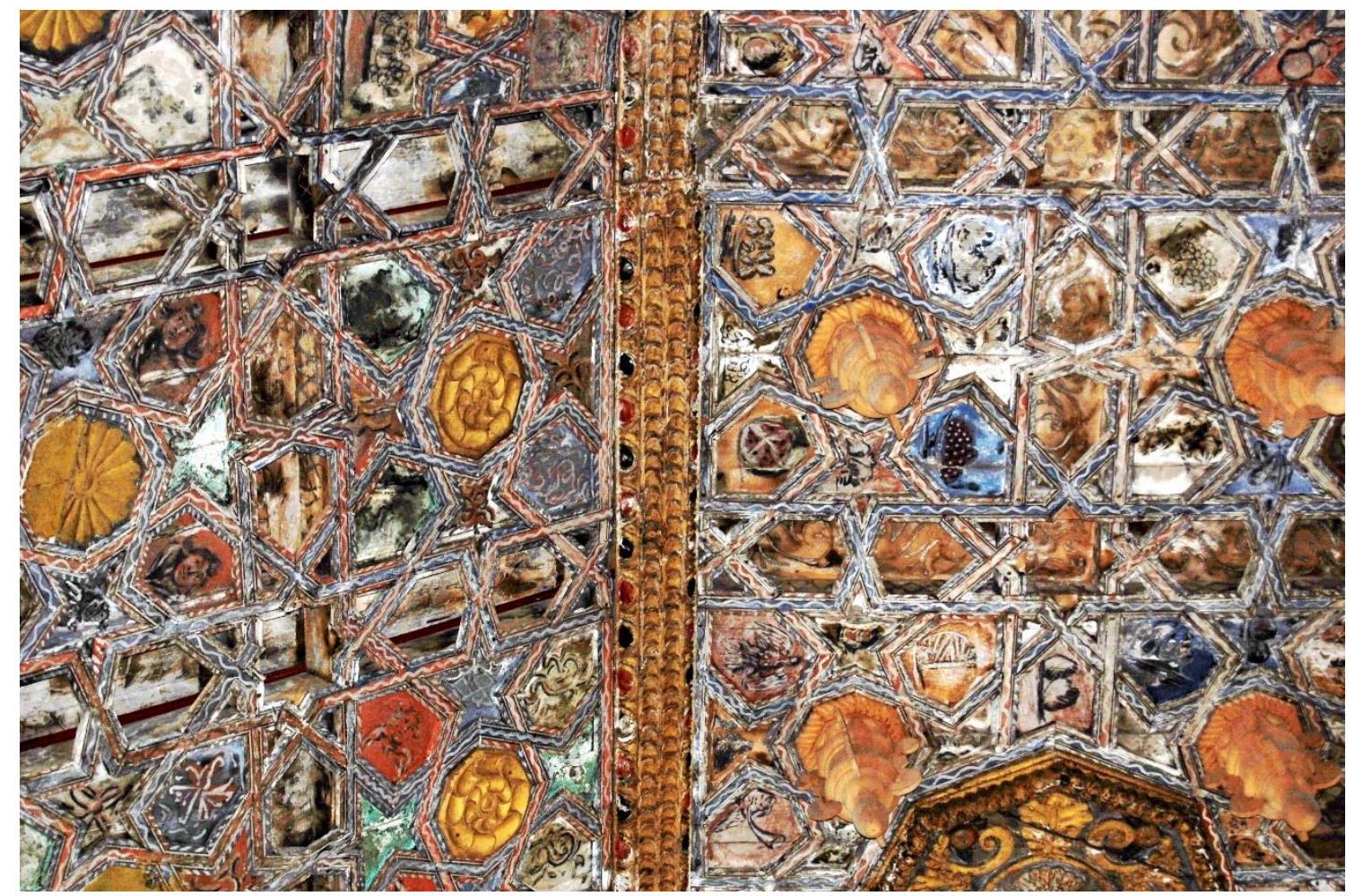

Foto 2. Detalle del faldón mayor y el almizate con variada representación iconográfica.

\section{METODOLOGÍA}

Las seis muestras, preparadas en forma de secciones transversales de corte pulido, se remitieron al Laboratorio de Análisis Físico-Químico y Control Medioambiental del Instituto Universitario de Restauración del Patrimonio (UPV) para que -partiendo de ellas- se hiciera el análisis. El estudio estratigráfico se realizó con un Microscopio Óptico Leica DMR con un sistema de luz incidente/transmitida y un sistema de polarización en ambos casos. Tras preparar la muestra, con el propósito de caracterizar morfológicamente las mismas, se ha llevado a cabo un estudio mediante microscopía óptica de sus correspondientes secciones transversales de corte pulido. Se realiza un análisis cualitativo mediante Microscopía Electrónica de Barrido con Espectrometría de rayos-X (SEM/EDX), es decir, la información a obtener es la identificación de los materiales constituyentes de la obras. Para ello, dichas muestras preparadas en sección transversal se recubren con carbono grafito.

\footnotetext{
${ }^{6}$ Marrero Alberto, A. (2012). Artesonado de la Capilla Mayor del Santuario del Santísimo Cristo de
} Tacoronte: Desarrollo Iconográfico y su Interpretación. Revista de Historia Canaria (193), pp. 89-122. 


\section{Microscopio electrónico de barrido y elucubraciones históricas. El artesonado mudéjar del santuario de Tacoronte (Tenerife)}

Seguidamente se adquieren los espectros de rayos-X en áreas o zonas puntuales de las mismas, seleccionadas estas por la presencia destacada de gránulos de mineral y la de pigmento finamente molido y aglutinado.

Debido a las considerables dimensiones de la obra, los criterios para la toma de muestras no se efectuaron en zonas poco visibles o distales, como podría ocurrir en una obra de menor formato, sino que el objetivo era contar con ejemplos de los colores presentes en el artesonado: blanco, amarillo, rojo, verde, azul claro y azul muy oscuro, para identificar su composición química y determinar el pigmento utilizado.

\section{RESULTADOS}

El análisis, mediante microscopio óptico, señaló la ausencia de barniz o capa de protección, que se justifica por las dimensiones del artesonado, su difícil acceso (imposible si no se accede desde el interior) y su eclipsamiento por la escultura del Cristo que recibiría todas las atenciones, pasando la techumbre a un segundo plano. Sin embargo, y aunque las representaciones sean en ocasiones tremendamente simplistas o se haya hecho empleo de un pincel grueso de forma rápida, con pinceladas anchas y largas, el estudio detallado que permite el SEM/EDX, conjuntamente con el desarrollo iconográfico, pone en valor este techo, ejemplo paradigmático dentro de los artesonados canarios.

Los resultados obtenidos por cada una de las muestras, incluyendo su interpretación, serían los siguientes:

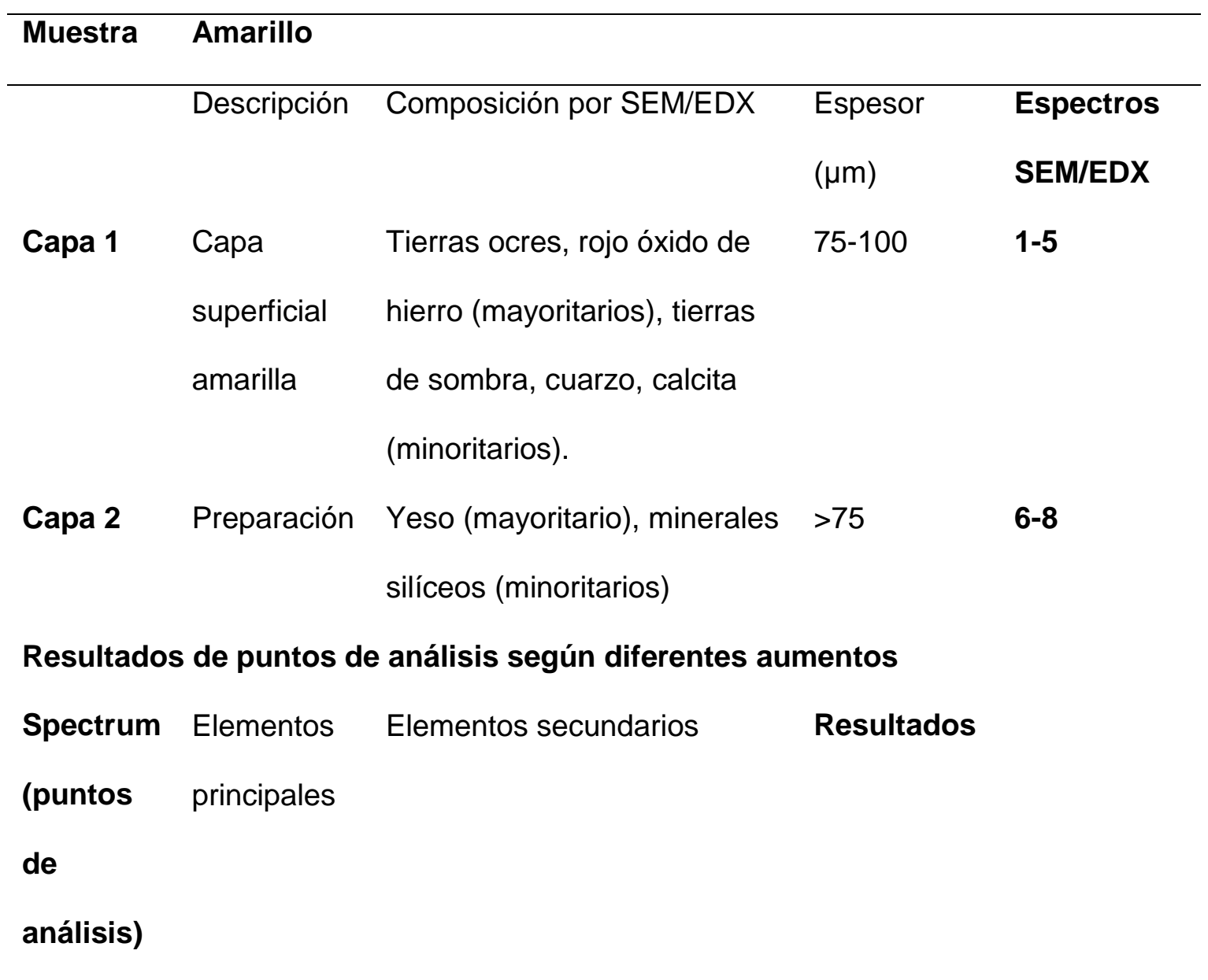


Microscopio electrónico de barrido y elucubraciones históricas. El artesonado mudéjar del santuario de Tacoronte (Tenerife)

\begin{tabular}{|c|c|c|c|}
\hline \multicolumn{4}{|c|}{ GEN, BKS, X70 } \\
\hline \multirow[t]{3}{*}{1} & $29.34 \%$ & $48.40 \% \mathrm{SiO}_{2}, 8.30 \% \mathrm{Al}_{2} \mathrm{O}_{3}$ & Ocre amarillo e \\
\hline & $\mathrm{FeO}$ & $6.79 \% \mathrm{SO}_{3}, 4.30 \% \mathrm{CaO}$ & impurezas de \\
\hline & & $1.43 \% \mathrm{~K}_{2} \mathrm{O}, 1.43 \% \mathrm{TiO}_{2}$ & $\begin{array}{l}\text { componentes arcillosos y } \\
\text { terrosos }\end{array}$ \\
\hline 2 & $1.90 \% \mathrm{FeO}$ & $53.90 \% \mathrm{SO}_{3}, 34.34 \% \mathrm{CaO}$ & Sulfato cálcico, empleado \\
\hline & & $7.24 \% \mathrm{SiO}_{2}, 2.62 \% \mathrm{Al}_{2} \mathrm{O}_{3}$ & $\begin{array}{l}\text { como carga en la capa de } \\
\text { preparación }\end{array}$ \\
\hline
\end{tabular}

DET PICT, BKS, X350

1

\begin{abstract}
$66.59 \%$
\end{abstract}
$28.41 \% \mathrm{SiO}_{2}, 3.69 \% \mathrm{Al}_{2} \mathrm{O}_{3}$,

Ocre amarillo e

$\mathrm{FeO}$

$1.31 \% \mathrm{CaO}$

impurezas (pertenece a la

zona superficial externa)

2

$49.03 \%$

$39.52 \% \mathrm{SiO}_{2}, 7.47 \% \mathrm{Al}_{2} \mathrm{O}_{3}$,

Ocre amarillo e

$\mathrm{FeO}$

$1.77 \% \mathrm{TiO}_{2}, 1.13 \mathrm{CaO}, 1.08$

impurezas

$\mathrm{K}_{2} \mathrm{O}$

3

$$
28.11 \%
$$

$27.35 \% \mathrm{SO}_{3}, 19.06 \% \mathrm{CaO}$,

Ocre amarillo, sulfato

$\mathrm{FeO}$

$17.24 \% \mathrm{SiO}_{2}, 7.47 \% \mathrm{Al}_{2} \mathrm{O}_{3}$,

cálcico (empleado como

$0.78 \% \mathrm{~K}_{2} \mathrm{O}$

carga en la capa de

preparación) e impurezas

4

$24.58 \%$

$66.45 \% \mathrm{SiO}_{2}, 7.06 \% \mathrm{Al}_{2} \mathrm{O}_{3}$,

Ocre amarillo e

$\mathrm{FeO}$

$1.91 \% \mathrm{CaO}$

impurezas

5

$65.92 \%$
$\mathrm{FeO}$

6

$23.60 \% \mathrm{SiO}_{2}, 4.88 \% \mathrm{MnO}$,

Ocre amarillo e

$2.95 \% \mathrm{Al}_{2} \mathrm{O}_{3}, 1.21 \% \mathrm{CaO}$,

impurezas

$0.83 \% \mathrm{TiO}_{2}, 0.51 \% \mathrm{~K}_{2} \mathrm{O}$

$59.69 \% \mathrm{SO}_{3}, 40.31 \% \mathrm{CaO}$

Sulfato cálcico, empleado

como carga en la capa de 
Microscopio electrónico de barrido y elucubraciones históricas. El artesonado mudéjar del santuario de Tacoronte (Tenerife)

8

$34.29 \%$

$\mathrm{FeO}$
$59.44 \% \mathrm{SO}_{3}, 39.21 \% \mathrm{CaO}$,

$1.35 \% \mathrm{SiO}_{2}$

$49.62 \% \mathrm{SiO}_{2}, 7.35 \% \mathrm{Al}_{2} \mathrm{O}_{3}$,

$4.42 \% \mathrm{SO}_{3}, 2.96 \% \mathrm{CaO}$,

$1.36 \% \mathrm{~K}_{2} \mathrm{O}$ preparación

Sulfato cálcico, empleado

como carga en la capa de

preparación

Ocre amarillo e

impurezas

DET PICT, BKS, X350 2

1

$\begin{array}{lll}65.71 \% & 23.43 \% \mathrm{SiO}_{2}, 4.54 \% \mathrm{MnO}, & \text { Ocre amarillo e } \\ \mathrm{FeO} & 2.13 \% \mathrm{Al}_{2} \mathrm{O}_{3}, 1.75 \% \mathrm{P}_{2} \mathrm{O}_{5}, & \text { impurezas } \\ & 1.70 \% \mathrm{CaO}, 0.66 \% \mathrm{~K}_{2} \mathrm{O} & \end{array}$

2

$79.63 \%$

$17.62 \% \mathrm{SiO}_{2}, 2.05 \% \mathrm{Al}_{2} \mathrm{O}_{3}$,

Ocre amarillo e

$\mathrm{FeO}$

$0.70 \% \mathrm{CaO}$

impurezas

3

$100 \% \mathrm{SiO}_{2}$

Impurezas aglutinadas

con el pigmento

4

$$
21.90 \%
$$

$31.94 \% \mathrm{SO}_{3}, 20.99 \% \mathrm{SiO}_{2}$,

Ocre amarillo e

$$
\mathrm{FeO}
$$

$19.23 \% \mathrm{CaO}, 5,93 \% \mathrm{Al}_{2} \mathrm{O}_{3}$

impurezas

5

$35.01 \%$

$\mathrm{FeO}$

$39.75 \% \mathrm{SiO}_{2}, 14.97 \% \mathrm{Al}_{2} \mathrm{O}_{3}$,

$4.38 \% \mathrm{SO}_{3}, 2.60 \% \mathrm{CaO}$,

Ocre amarillo e

$1.99 \% \mathrm{~K}_{2} \mathrm{O}, 1.29 \% \mathrm{TiO}_{2}$

6

$59.83 \% \mathrm{SO}_{3}, 40.17 \% \mathrm{CaO}$

Sulfato cálcico, empleado

como carga en la capa de

preparación

7

$61.48 \% \mathrm{SO}_{3}, 38.52 \% \mathrm{CaO}$

Sulfato cálcico, empleado como carga en la capa de preparación 
Microscopio electrónico de barrido y elucubraciones históricas. El artesonado mudéjar del santuario de Tacoronte (Tenerife)

8

9

10
$60.59 \% \mathrm{SO}_{3}, 39.41 \% \mathrm{CaO} \quad$ Sulfato cálcico, empleado

como carga en la capa de

preparación

$60.39 \% \mathrm{SO}_{3}, 39.61 \% \mathrm{CaO} \quad$ Sulfato cálcico, empleado

como carga en la capa de

preparación

Sulfato cálcico, empleado

como carga en la capa de

preparación

La capa pictórica presenta un espesor $75-100 \mu \mathrm{m}$ y constituida por pigmentos tipo tierras ocre y rojo óxido de hierro (componentes mayoritarios) y exhibe algunos componentes minoritarios del tipo tierras de sombra, cuarzo y calcita.

Muestra Azul claro

Descripción $\quad$ Composición por SEM/EDX Espesor $\quad$ Espectros

$(\mu \mathrm{m}) \quad$ SEM/EDX

Capa 1 Capa Azul esmalte, tierras, calcita, $\quad 130-175 \quad \mathbf{1 - 4}$ y

superficial pigmento de plomo distribució

azul n n

puntual

Capa 2 Preparación -

Resultados de puntos de análisis según diferentes aumentos

Spectrum Elementos Elementos secundarios Resultados

(puntos principales

de

análisis)

GEN, BKS, X33 
Microscopio electrónico de barrido y elucubraciones históricas. El artesonado mudéjar del santuario de Tacoronte (Tenerife)

$\begin{array}{lll}1.78 \% \mathrm{CoO} & 75.66 \% \mathrm{SiO}_{2}, 9.01 \% \mathrm{~K}_{2} \mathrm{O}, & \text { Óxido de cobalto e } \\ 6.11 \% \mathrm{CaO}, 2.59 \% \mathrm{FeO}, & \text { impurezas } \\ & 2.53 \% \mathrm{As} 2 \mathrm{O} 3,2.31 \% \mathrm{Al}_{2} \mathrm{O}_{3} & \end{array}$

PICT, BKS, X140

1

2

3

4

5

6

$52.53 \%$

$\mathrm{PbO}$

7

$33.47 \%$

$\mathrm{PbO}$

8

$40.37 \%$

$\mathrm{PbO}$
$1.44 \% \mathrm{CoO}$

$5.28 \% \mathrm{CaO}, 2.13 \% \mathrm{As} 2 \mathrm{O} 3$,

$1.59 \% \mathrm{Al}_{2} \mathrm{O}_{3}, 1.50 \% \mathrm{FeO}$

$1.18 \% \mathrm{CoO}$

$5.63 \% \mathrm{CaO}, 2.26 \% \mathrm{As}_{2} \mathrm{O}_{3}$,

$1.63 \% \mathrm{Al}_{2} \mathrm{O}_{3}, 1.43 \% \mathrm{FeO}$

$74.23 \% \mathrm{SiO}_{2}, 17.85 \% \mathrm{~K}_{2} \mathrm{O}$,

$5.55 \% \mathrm{CaO}, 2.37 \% \mathrm{FeO}$

$82.83 \% \mathrm{SiO}_{2}, 8.56 \% \mathrm{CaO}$,

$3.37 \% \mathrm{SO}_{3}, 2.76 \% \mathrm{As}_{2} \mathrm{O}_{3}$,

$2.48 \% \mathrm{Al}_{2} \mathrm{O}_{3}$

$83.61 \% \mathrm{SO} 3,8.27 \% \mathrm{~K}_{2} \mathrm{O}$,

$8.13 \% \mathrm{CaO}$

$24.34 \% \mathrm{~K}_{2} \mathrm{O}, 14.64 \% \mathrm{As}_{2} \mathrm{O}_{3}$

$21.24 \% \mathrm{CaO}, 20.53 \% \mathrm{~K}_{2} \mathrm{O}$,

$14.06 \% \mathrm{SO}_{3}, 10.69 \% \mathrm{SiO}_{2}$

$26.26 \% \mathrm{CaO}, 17.77 \% \mathrm{SiO}_{2}$,

$15.61 \% \mathrm{~K}_{2} \mathrm{O}$ como carga en la capa de

preparación

Blanco de plomo e

impurezas aglutinadas

con el pigmento

Impurezas aglutinadas

con el pigmento

Impurezas aglutinadas

con el pigmento

Sulfato cálcico, empleado

Blanco de plomo e

impurezas aglutinadas

con el pigmento

Blanco de plomo e

impurezas aglutinadas 
Microscopio electrónico de barrido y elucubraciones históricas. El artesonado mudéjar del santuario de Tacoronte (Tenerife)

con el pigmento

9

$$
\begin{aligned}
& 6.46 \% \mathrm{CaO}, 2.77 \% \mathrm{FeO}, \\
& 2.72 \% \mathrm{Al}_{2} \mathrm{O}_{3}
\end{aligned}
$$

Óxido de cobalto e

impurezas aglutinadas

con el pigmento

PICT, BKS, X500

Blanco de plomo e

7.37\% FeO, 7.13\% $\mathrm{CaO}$, impurezas aglutinadas

$4.88 \% \mathrm{Al}_{2} \mathrm{O}_{3}, 3.64 \% \mathrm{SO} 3$, con el pigmento

$3.09 \% \mathrm{MgO}, 2.25 \% \mathrm{~K}_{2} \mathrm{O}$

2

$$
27.78 \%
$$

$22.65 \% \mathrm{SiO}_{2}, 15.08 \% \mathrm{SO}$,

Blanco de plomo e

$\mathrm{PbO}$

$13.74 \% \mathrm{CaO}, 9.64 \% \mathrm{As}_{2} \mathrm{O}_{3}$,

$3.65 \% \mathrm{FeO}, 3.42 \% \mathrm{~K}_{2} \mathrm{O}$,

impurezas aglutinadas

$2.25 \% \mathrm{Al}_{2} \mathrm{O}_{3}$

3

$$
37.24 \%
$$

$31.55 \% \mathrm{CaO}, 13.69 \% \mathrm{SiO}_{2}$,

$\mathrm{PbO}$

$4.90 \% \mathrm{~K}_{2} \mathrm{O}, 4.75 \% \mathrm{P}_{2} \mathrm{O}_{5}$,

$3.27 \% \mathrm{FeO}, 1.27 \% \mathrm{Al}_{2} \mathrm{O}_{3}$

4

$59.57 \%$

$21.08 \% \mathrm{~K}_{2} \mathrm{O}, 19.35 \% \mathrm{CaO}$

$\mathrm{PbO}$ con el pigmento

Blanco de plomo e

impurezas aglutinadas

con el pigmento

Blanco de plomo e

impurezas aglutinadas

con el pigmento

Tabla 2. Muestra estratigráfica azul 2 (azul claro).

La capa pictórica presenta un espesor 130-175 $\mu \mathrm{m}$ y está constituida por el pigmento azul esmalte (aluminosilicato de cobalto) y granos aislados de tierras (Silicatos de aluminio y hierro) y calcita $\left(\mathrm{CaCO}_{3}\right)$. Se identifica también la presencia de plomo $(\mathrm{Pb})$ asociado a un pigmento de plomo. La capa de preparación está constituida principalmente por yeso. 
Microscopio electrónico de barrido y elucubraciones históricas. El artesonado mudéjar del santuario de Tacoronte (Tenerife)

\begin{tabular}{lllll}
\hline Muestra & Azul oscuro & & & \\
& & & & \\
& & & & \\
& & & $(\mu \mathrm{m})$ & SEM/EDX \\
Capa 1 & Capa azul & Azul esmalte (mayoritario), & $120-130$ & $\mathbf{1 - 3}$ \\
& oscuro & tierras, yeso (minoritario) & & \\
Capa 2 & Preparación & Yeso (mayoritario), minerales & $>450$ & $\mathbf{4 , 5}$ \\
& Blanca & silíceos, calcita & &
\end{tabular}

Resultados de puntos de análisis según diferentes aumentos

Spectrum Elementos Elementos secundarios Resultados

(puntos principales

de

análisis)

GEN, BKS, X23

1

48.62\% SO3, 29.77\% CaO, Sulfato cálcico, empleado

$21.61 \% \mathrm{SiO}_{2}$

como carga en la capa de

preparación, con trazas

de impurezas

GEN, BKS, X23 2

1

$2.12 \% \mathrm{CoO}$

$72.39 \% \mathrm{SiO}_{2}, 14.46 \% \mathrm{~K}_{2} \mathrm{O}$,

Óxido de cobalto e

$5.19 \% \mathrm{CaO}, 1.95 \% \mathrm{FeO}$,

impurezas

$1.57 \% \mathrm{As}_{2} \mathrm{O}_{3}, 1.43 \% \mathrm{Al}_{2} \mathrm{O}_{3}$

$0.88 \% \mathrm{NiO}$

2

$61.13 \% \mathrm{SO} 3,38.87 \% \mathrm{CaO}$

Sulfato cálcico, empleado

como carga en la capa de

preparación

DET PICT, BKS, X130 
Microscopio electrónico de barrido y elucubraciones históricas. El artesonado mudéjar del santuario de Tacoronte (Tenerife)

$1.90 \% \mathrm{CoO} \quad 69.20 \% \mathrm{SiO}_{2}, 16.62 \% \mathrm{~K}_{2} \mathrm{O}, \quad$ Óxido de cobalto e

$6.22 \% \mathrm{CaO}, 1.96 \% \mathrm{FeO}, \quad$ impurezas

$1.55 \% \mathrm{As}_{2} \mathrm{O}_{3}, 1.50 \% \mathrm{Al}_{2} \mathrm{O}_{3}$,

$1.06 \% \mathrm{NiO}$

2

$2.57 \% \mathrm{CoO}$

$80.81 \% \mathrm{SiO}_{2}, 10.05 \% \mathrm{~K}_{2} \mathrm{O}$

Óxido de cobalto e

$2.16 \% \mathrm{FeO}, 1.21 \% \mathrm{Al}_{2} \mathrm{O}_{3}$,

impurezas

$1.13 \%$ As2O3, $1.11 \% \mathrm{CaO}$,

$0.96 \% \mathrm{NiO}$

3

$44.04 \% \mathrm{SO} 3,38.50 \% \mathrm{CaO}$,

Sulfato cálcico, empleado

$7.23 \% \mathrm{SiO}_{2}, 2.26 \% \mathrm{TiO}_{2}$,

como carga en la capa de

$2.18 \% \mathrm{~K}_{2} \mathrm{O}, 2.05 \% \mathrm{FeO}$,

preparación, e impurezas

$1.47 \% \mathrm{Al}_{2} \mathrm{O}_{3}$

4

$58.96 \%$ SO3, $40.03 \% \mathrm{CaO}$,

Sulfato cálcico, empleado

$1.02 \% \mathrm{SiO}_{2}$

como carga en la capa de

preparación

5

$60.15 \%$ SO3, 39.85\% CaO

Sulfato cálcico, empleado

como carga en la capa de

preparación

6

$2.27 \% \mathrm{CoO}$

$82.51 \% \mathrm{SiO}_{2}, 6.33 \% \mathrm{CaO}$,

Óxido de cobalto e

$2.58 \% \mathrm{FeO}, 2.13 \% \mathrm{Al}_{2} \mathrm{O}_{3}$,

impurezas

$1.93 \% \mathrm{As}_{2} \mathrm{O}_{3}, 1.16 \% \mathrm{NiO}$,

$1.08 \% \mathrm{~K}_{2} \mathrm{O}$

Tabla 3. Muestra estratigráfica azul (azul oscuro)

La capa pictórica presenta un espesor 120-130 $\mu \mathrm{m}$ y está constituida por el pigmento azul esmalte (aluminosilicato de cobalto) y granos aislados de tierras. La capa de preparación está constituida principalmente por yeso $(\mathrm{CaSO} 4.2 \mathrm{H} 2 \mathrm{O})$ y minerales silíceos accesorios. Las distribuciones puntuales de elementos obtenidas confirman estos resultados. 
Microscopio electrónico de barrido y elucubraciones históricas. El artesonado mudéjar del santuario de Tacoronte (Tenerife)

\begin{tabular}{|c|c|c|c|c|}
\hline Muestra & Rojo & & & \\
\hline & Descripción & Composición por SEM/EDX & $\begin{array}{l}\text { Espesor } \\
(\mu \mathrm{m})\end{array}$ & $\begin{array}{l}\text { Espectros } \\
\text { SEM/EDX }\end{array}$ \\
\hline Capa 1 & $\begin{array}{l}\text { Capa } \\
\text { pictórica } \\
\text { superficial } \\
\text { roja }\end{array}$ & $\begin{array}{l}\text { Minio (mayoritario), minerales } \\
\text { silíceos (minoritario) }\end{array}$ & $20-25$ & $1-3$ \\
\hline Capa 2 & $\begin{array}{l}\text { Preparación } \\
\text { Blanca }\end{array}$ & $\begin{array}{l}\text { Yeso (mayoritario), minerales } \\
\text { silíceos, celestina, pigmento } \\
\text { de plomo (minoritarios) }\end{array}$ & $>290$ & $\begin{array}{l}\text { 4-8 y } \\
\text { distribució } \\
\text { n puntual }\end{array}$ \\
\hline Resultados & de puntos $\mathrm{d}$ & análisis según diferentes au & nentos & \\
\hline $\begin{array}{l}\text { Spectrum } \\
\text { (puntos } \\
\text { de } \\
\text { análisis) }\end{array}$ & $\begin{array}{l}\text { Elementos } \\
\text { principales }\end{array}$ & Elementos secundarios & Resultac & \\
\hline GEN, BK & X75 & & & \\
\hline 1 & $\begin{array}{l}90.92 \% \\
\mathrm{PbO}\end{array}$ & - & Rojo de & \\
\hline 2 & $100 \% \mathrm{PbO}$ & - & Rojo de & \\
\hline 3 & $7.24 \% \mathrm{PbO}$ & $\begin{array}{l}54.69 \% \mathrm{SO}, 36.06 \% \mathrm{CaO}, \\
1.14 \% \mathrm{SiO}_{2}, 0.97 \% \mathrm{Al}_{2} \mathrm{O}_{3}\end{array}$ & $\begin{array}{l}\text { Sulfato } \\
\text { como ca } \\
\text { prepara } \\
\text { trazas d } \\
\text { de la car }\end{array}$ & $\begin{array}{l}\text { co, empleado } \\
\text { en la capa de } \\
\text { presenta } \\
\text { mo migrado } \\
\text { ctórica }\end{array}$ \\
\hline
\end{tabular}


Microscopio electrónico de barrido y elucubraciones históricas. El artesonado mudéjar del santuario de Tacoronte (Tenerife)

DET PICT, BKS, X330

$61.01 \% \mathrm{SO}_{3}, 38.99 \% \mathrm{CaO} \quad$ Sulfato cálcico, empleado

como carga en la capa de

preparación

2

$11.85 \%$

$53.43 \% \mathrm{SO}_{3}, 34.72 \% \mathrm{CaO}$

Sulfato cálcico, empleado

$\mathrm{PbO}$

como carga en la capa de

preparación, presenta

trazas de plomo migrado

de la capa pictórica

3

$20.40 \%$

$45.21 \% \mathrm{SO}_{3}, 34.40 \% \mathrm{CaO}$

Sulfato cálcico, empleado

$\mathrm{PbO}$

como carga en la capa de

preparación, presenta

trazas de plomo migrado

de la capa pictórica

4

$54.58 \% \mathrm{SO}_{3}, 41.72 \% \mathrm{CaO}$,

Sulfato cálcico, empleado

$1.85 \% \mathrm{SiO}_{2}, 1.85 \% \mathrm{FeO}$

como carga en la capa de

preparación

5

$81.04 \%$

Rojo de plomo

$\mathrm{PbO}$

6

$100 \% \mathrm{SiO}_{2}$

Impurezas aglutinadas

junto con el pigmento

7

$92.53 \%$

$4.66 \% \mathrm{CaO}, 2.81 \% \mathrm{Al}_{2} \mathrm{O}_{3}$

Rojo de plomo

$\mathrm{PbO}$

8

Sulfato cálcico, empleado

$\mathrm{PbO}$

como carga en la capa de

preparación, presenta 
Microscopio electrónico de barrido y elucubraciones históricas. El artesonado mudéjar del santuario de Tacoronte (Tenerife)

trazas de plomo migrado

de la capa pictórica

Sulfato cálcico, empleado

como carga en la capa de

preparación, presenta

trazas de plomo migrado

de la capa pictórica

DET PICT, BKS, X180

1

2

3

4

5
$45.81 \%$ SO3, $54.19 \%$ SrO

$36.27 \% \mathrm{SO}_{3}, 24.88 \% \mathrm{CaO}$

$\mathrm{PbO}$

$58.51 \% \mathrm{SO}_{3}, 41.49 \% \mathrm{CaO}$

$46.52 \% \mathrm{SO}_{3}, 29.70 \% \mathrm{CaO}$,

$5.93 \% \mathrm{SiO}_{2}, 3.99 \% \mathrm{Al}_{2} \mathrm{O}_{3}$

$1.71 \mathrm{NiO}, 1.06 \% \mathrm{TiO}_{2}, 0.99 \%$

$\mathrm{K}_{2} \mathrm{O}, 0.84 \% \mathrm{MgO}$

$8.39 \% \mathrm{PbO}$

$5.59 \% \mathrm{PbO}$
$55.24 \% \mathrm{SO}_{3}, 37.20 \% \mathrm{CaO}$,

$1.31 \% \mathrm{SiO}_{2}, 0.65 \% \mathrm{Al}_{2} \mathrm{O}_{3}$
Sulfato de estroncio

Sulfato cálcico, empleado

como carga en la capa de

preparación, presenta

trazas de plomo migrado

de la capa pictórica

Sulfato cálcico, empleado

como carga en la capa de

preparación

Sulfato cálcico, empleado como carga en la capa de

preparación, presenta

trazas de plomo migrado

de la capa pictórica, e

impurezas

Sulfato cálcico, empleado como carga en la capa de preparación, presenta 
Microscopio electrónico de barrido y elucubraciones históricas. El artesonado mudéjar del santuario de Tacoronte (Tenerife)

trazas de plomo migrado

de la capa pictórica

Tabla 4. Muestra estratigráfica roja

La capa pictórica presenta un espesor entre 20-25 $\mu \mathrm{m}$ y presenta cierta tonalidad roja que indican la presencia de minio como pigmento (Pb3O4) y granos aislados de tierras (minerales silíceos). El estrato preparatorio presenta yeso, aunque, en los análisis puntuales realizados se manifiesta también la presencia significativa de minerales silíceos y celestina (variedad mineral de sulfato de estroncio), como minerales accesorios del yeso y pigmento de plomo.

\begin{tabular}{lllll}
\hline Muestra & Verde & & & \\
\hline & Descripción & Composición por SEM/EDX & Espesor & Espectros \\
& & & $(\mu \mathrm{m})$ & SEM/EDX \\
Capa 1 & Capa verde & Pigmento de cobre, tierras, & $25-60$ & $\mathbf{1 - 2}$ \\
& & calcita, blanco de plomo & \\
Capa 2 & Preparación & Yeso (mayoritario), minerales & $>240$ & $\mathbf{3 - 5}$ \\
& Blanca & silíceos, calcita & &
\end{tabular}

Resultados de puntos de análisis según diferentes aumentos

Spectrum Elementos Elementos secundarios Resultados

(puntos principales

de

análisis)

GEN, BKS, X60

1

$83.57 \%$

$7.86 \% \mathrm{SO} 3,4.23 \% \mathrm{CaO}$

Óxido de cobre (verde

$\mathrm{CuO}$

malaquita)

2

$3.72 \% \mathrm{CuO} \quad 55.25 \% \mathrm{SO}, 41.03 \% \mathrm{CaO}$

Sulfato cálcico, empleado

como carga en la capa de

preparación, presenta

trazas de cobre migrado 
Microscopio electrónico de barrido y elucubraciones históricas. El artesonado mudéjar del santuario de Tacoronte (Tenerife)

de la capa pictórica

DET, BKS, X370

1

2

$91.38 \%$

$2.35 \% \mathrm{SO}, 1.28 \% \mathrm{CaO}$

Óxido de cobre (verde

$\mathrm{CuO}$

malaquita)

3

$86.69 \%$

$3.05 \% \mathrm{SO} 3,2.48 \% \mathrm{SiO}_{2}$

Óxido de cobre (verde

$\mathrm{CuO}$

$1.80 \% \mathrm{Al}_{2} \mathrm{O}_{3}, 1.32 \% \mathrm{CaO}$

malaquita)

4

$100 \% \mathrm{SiO}_{2}$

Impurezas aglutinadas

junto con el pigmento

5

$90.04 \%$

$2.30 \% \mathrm{SO} 3,1.12 \% \mathrm{CaO}$

Óxido de cobre (verde

$\mathrm{CuO}$

malaquita)

6

$6.09 \% \mathrm{CuO} \quad 57.67 \% \mathrm{SO} 3,36.24 \% \mathrm{CaO}$

Sulfato cálcico, empleado

como carga en la capa de

preparación, presenta

trazas de cobre migrado

de la capa pictórica

7

$5.31 \% \mathrm{CuO} \quad 55.07 \% \mathrm{SO} 3,33.61 \% \mathrm{CaO}$,

Sulfato cálcico, empleado

$4.51 \% \mathrm{SiO}_{2}, 1.50 \% \mathrm{Al}_{2} \mathrm{O}_{3}$

como carga en la capa de

preparación, presenta

trazas de plomo migrado

de la capa pictórica, e

impurezas

8

$3.00 \% \mathrm{CuO} \quad 45.36 \% \mathrm{SiO}_{2}, 21.35 \% \mathrm{MgO}$,

Impurezas con trazas de

$14.43 \% \mathrm{SO}, 9.02 \% \mathrm{CaO}$

Sulfato cálcico de la

$2.71 \% \mathrm{TiO}_{2}, 1.27 \% \mathrm{Al}_{2} \mathrm{O}_{3}$

preparación y óxido de

cobre del pigmento que 
Microscopio electrónico de barrido y elucubraciones históricas. El artesonado mudéjar del santuario de Tacoronte (Tenerife)

ha migrado

9

$19.68 \%$

CuO

44.26\% SO3, $25.85 \% \mathrm{CaO}$,

$7.02 \% \mathrm{SiO}_{2}, 1.50 \% \mathrm{Al}_{2} \mathrm{O}_{3}$,

$0.89 \% \mathrm{TiO}_{2}$
Sulfato cálcico, empleado

como carga en la capa de

preparación, presenta

trazas de plomo migrado

de la capa pictórica e

impurezas

Tabla 5. Muestra estratigráfica v erde

La capa pictórica presenta un espesor entre 25-60 $\mu \mathrm{m}$ y presenta cierta tonalidad verde, lo que indica la presencia de un pigmento de cobre. Los análisis puntuales y de área llevados a cabo en la capa de preparación confirman la presencia mayoritaria de yeso (CaSO4.2H2O). También se ha identificado la presencia de tierras y calcita, como minerales accesorios del yeso, y cobre, relativo al estrato pictórico.

Muestra Blanco

Descripción Composición por SEM/EDX Espesor Espectros

$(\mu \mathrm{m}) \quad$ SEM/EDX

Capa 1 Depósitos Tierras, calcita $\quad-\quad 3$,

superficiale

Distribució

S

n puntual

Capa 2

Capa Blanco de plomo

$>480$

$1-2,4$,

superficial (mayoritario), tierras, cuarzo

Distribució

Blanca

(minoritarios)

n puntual

Resultados de puntos de análisis según diferentes aumentos

Spectrum Elementos Elementos secundarios Resultados

(puntos principales 
Microscopio electrónico de barrido y elucubraciones históricas. El artesonado mudéjar del santuario de Tacoronte (Tenerife)

de

análisis)

GEN, BKS, X90

3

4

$100 \% \mathrm{PbO}$

Blanco de plomo

2

$100 \% \mathrm{PbO}$

Blanco de plomo

$100 \% \mathrm{PbO}$

Blanco de plomo

4

$100 \% \mathrm{PbO}$

Blanco de plomo

5

$100 \% \mathrm{PbO}$

Blanco de plomo

DET INF, BKS, X370$$
1
$$

2

3

4

5

$92.22 \%$

$7.78 \% \mathrm{Bi}_{2} \mathrm{O}_{3}$

$\mathrm{PbO}$

6

$100 \% \mathrm{PbO}$

Blanco de plomo

7

$100 \% \mathrm{PbO}$

Blanco de plomo

8

$98.04 \%$

$1.31 \% \mathrm{SiO}_{2}, 0.64 \% \mathrm{CaO}$

Blanco de plomo

$\mathrm{PbO}$

DET SUP, BKS, X600

1

$\begin{array}{ll}53.49 \% & 20.24 \% \mathrm{SiO}_{2}, 8.99 \% \mathrm{Al}_{2} \mathrm{O}_{3}, \\ \mathrm{PbO} & 8.19 \% \mathrm{CaO}, 7.96 \% \mathrm{FeO}, \\ & 1.13 \% \mathrm{~K}_{2} \mathrm{O}\end{array}$

$1.13 \% \mathrm{~K}_{2} \mathrm{O}$

2

$100 \% \mathrm{PbO}$

3

$100 \% \mathrm{PbO}$

la zona superficial

externa)

Blanco de plomo

Blanco de plomo y trazas

de suciedad (pertenece a

Blanco de plomo 
Microscopio electrónico de barrido y elucubraciones históricas. El artesonado mudéjar del santuario de Tacoronte (Tenerife)

4

56.5
$\mathrm{PbO}$

5

6

7

$27.22 \%$

$\mathrm{PbO}$

$97.52 \%$

$\mathrm{PbO}$

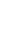

$27.35 \% \mathrm{FeO}, 9.15 \% \mathrm{TiO}_{2}$,

$3.44 \% \mathrm{SiO}_{2}, 1.75 \% \mathrm{Al}_{2} \mathrm{O}_{3}$,

$1.74 \mathrm{MgO}$
$100 \% \mathrm{SiO}_{2}$

$72.78 \% \mathrm{SiO}_{2}$
Blanco de plomo y trazas

de suciedad (pertenece a

la zona superficial

externa)

Blanco de plomo

Impurezas aglutinadas

con el pigmento

Blanco de plomo en

pequeña proporción, ya

que se analizó una zona

oscura que se deduce

que no pertenece al

conjunto granulométrico

propio del $\mathrm{PbO}$

Tabla 6. Muestra estratigráfica blanca

La superficie de esta muestra exhibe una fina capa de depósitos superficiales formados por tierras y calcita. La capa pictórica presenta un espesor mayor de $480 \mu \mathrm{m}$, y está constituida por el pigmento blanco de plomo $\left(2 \mathrm{PbCO}_{3} \cdot \mathrm{Pb}(\mathrm{OH})_{2}\right)$ y granos aislados de tierras (silicatos de aluminio y hierro).

Tras el análisis de las estratigrafías y teniendo en cuenta los elementos y compuestos inorgánicos detectados, los colores empleados en el artesonado tacorontero son los siguientes ${ }^{7}$ :

Ocre amarillo: es una arcilla coloreada por el hierro y que se presenta en varias tonalidades amarillas y apagadas. Es opaco y absolutamente permanente. Los grados mejores, más refinados y cuidadosamente lavados proceden de Francia. Su utilización se remonta a tiempos prehistóricos. Es un óxido de hierro muy estable a la témpera para tabla aunque no tiene fuerza suficiente como para aplicarse con total independencia. Sin

\footnotetext{
${ }^{7}$ Para su identificación se han empleado las siguientes fuentes: Vivancos Ramón, V. (2007). La conservación y restauración de pintura de caballete. Pintura sobre tabla. Madrid, España: Tecnos, pp. 85-99. Calvo, A. (1997). Conservación y restauración. Materiales, técnicas y procedimientos. De la $A$ a la
} 


\section{Antonio Marrero Alberto \\ Microscopio electrónico de barrido y elucubraciones históricas. El artesonado mudéjar del santuario de Tacoronte (Tenerife)}

embargo, aglutinado con aceite tiene un poder de cubrición más que notable, que le hace muy aconsejable para veladuras. En general, se emplea para aclarar los colores, hacer verdes, para aclarar las caras, o añadido a negro y blanco de plomo para crear la imprimación verdosa característica de los rostros de los primitivos italianos, llamado verdaccio. $^{8}$

Azul esmalte: una especie de cristal o frita azul cobalto, que se hace tostando un mineral de cobalto con otros ingredientes (silicato potásico coloreado con óxido de cobalto y luego triturado hasta quedar en polvo), de forma similar a como se hacía la frita azul egipcia; de hecho, se le considera históricamente como una continuación directa del color egipcio habiéndose sustituido el cobre por cobalto que es menos venenoso. Se ha encontrado cobalto entre los ingredientes de varias antiguas cerámicas azules, y también como impureza en azules de cobre, pero se considera que los pigmentos derivados de minerales de cobalto son una innovación nórdica. Durante su época de apogeo, antes de la introducción del ultramar artificial, el esmalte se preparaba cuidadosamente en varios grados, pero en la actualidad sólo se usa algo en cerámica y para pintar letreros.

Rojo de plomo o minio: se obtiene a partir de la cocción de minerales de plomo, dando origen a un óxido salino de plomo (tetróxido de plomo) de color anaranjado. Conocido también como minio, término empleado durante la Edad Media; anteriormente los romanos lo habían aplicado al cinabrio, su bermellón natural, y en menor grado a un óxido refinado. Quizás debido a que el cinabrio estaba con frecuencia adulterado con rojo de plomo, se le fue aplicando gradualmente el término minimum, que se convirtió en minium y acabó aplicándose al rojo de plomo. La palabra "miniatura" se deriva del uso de este pigmento en la iluminación de manuscritos. También se utilizaba para preparaciones o tonos de base y como color naranja. ${ }^{9}$

Verde malaquita (o verde montaña): $\mathrm{CuCO}_{3} \mathrm{Cu}(\mathrm{OH})_{2}$ carbonato básico de cobre. Tiene origen mineral, y está estrechamente ligado a la azurita. Se trata de un verde muy pálido y débil de tono, que se extraía de la piedra malaquita, el cual, por ser un pigmento muy granuloso debido a su molido, se usaba para bases y zonas planas y con poca relevancia, y no para veladuras. Sus propiedades son bastante similares a las de la azurita, aunque cuando se la aglutina con aceite se torna en un verde amarillento ${ }^{10}$.

Blanco de plomo: el blanco de plomo, albayalde o cerusa, es un carbonato básico de plomo $\left[2 \mathrm{PbCO}_{3} \cdot \mathrm{Pb}(\mathrm{OH})_{2}\right]$, y uno de los colores básicos de la pintura antigua y especialmente sobre tabla, pues por su calidad se solía aplicar solo o mezclado con otros para aclararlos. Se extraía de la costra originada a partir de exponer a los vapores del vinagre planchas de plomo y ni que decir tiene que durante su preparación se corría riesgo de envenenamiento sino se seguían las medidas necesarias. Este color se ha caracterizado por su gran versatilidad una vez aglutinado con un médium tanto en témpera como en aceite $^{11}$.

\section{DISCUSIÓN}

Aparte de la consabida función de las técnicas analíticas en cuanto al conocimiento matérico (elementos, pigmentos, cargas, aglutinantes, etc.) de las muestras a analizar, su estudio en profundidad y pormenorizado permite a los historiadores del arte una serie de reflexiones en torno a la obra y su creación, la cual sería imposible sin esta herramienta

\footnotetext{
8 Cennino, C. (1957): El libro del arte. Buenos Aires, Argentina: Argos, pp. 55-56.

${ }^{9}$ Cennino, C. (1957): El libro del arte. Buenos Aires, Argentina: Argos, p. 53.

10 Cennino, C. (1957): El libro del arte. Buenos Aires, Argentina: Argos, pp. 59-60.

11 Cennino, C. (1957): El libro del arte. Buenos Aires, Argentina: Argos, p. 63.
} 


\section{Antonio Marrero Alberto \\ Microscopio electrónico de barrido y elucubraciones históricas. El artesonado mudéjar del santuario de Tacoronte (Tenerife)}

científica. En el caso de estudio que nos ocupa se pueden llegar a las siguientes conclusiones:

El uso del ocre amarillo responde a la naturaleza propia de las Islas. Estas, de origen volcánico, son ricas en tierras férricas y de variados colores, con predominio de ocres, amarillos y anaranjados. No es extraño que este color sea el exponente de tierra natural obtenida del propio entorno, otorgando luz a la techumbre, junto con los numerosos elementos dorados y las corlas que jalonan dicha armadura.

De obtención complicada, los colores con base de plomo (blanco y rojo) son famosos por su poder de envenenamiento y la alta tasa de mortalidad de sus realizadores. Ambos tienen sustitutos más baratos, véase la laca roja (procedente de la cochinilla) o el carbonato cálcico para el blanco, el cual es mucho más barato y fácil de conseguir. En el caso del blanco de plomo, su uso para aclarar el azul de cobalto resulta llamativo, pues habiendo sustitutivos como el mencionado, decantarse por este blanco supone un gasto extra. Que se opten por estos dos colores de naturaleza plúmbica, tan peligrosos como caros, habla de una intencionalidad animista.

El Verde malaquita es, tal vez, el más inestable de los colores empleados, pues de fácil lavado por las filtraciones, hecho que se hace evidente en el artesonado que es objeto de nuestro estudio, donde dicho tono se ha perdido en la mayoría de los casos, arrastrado por el agua de la lluvia.

Tal vez sea este el caso en el que se hace más evidente que la intencionalidad propia de su policromía no es la meramente figurativa. El azul cobalto aparece en dos casos: mezclado con blanco de plomo para conseguir el azul celeste y sin mezclar para para una tonalidad negra (no se hace evidente que es un azul extremadamente oscuro hasta que no se observan con detalle los análisis). Tal vez el azul de cobalto no sea el más caro entre los pigmentos asociados a este color, siendo el máximo triunfador el azul ultramar, uno de los más costosos existentes, pero sí tiene una característica que lo identifica y es que su poder de tinción es tal, que basta con una mínima cantidad para conseguir un azul intenso. Es por ello que en la mayoría de los casos, el microscopio electrónico de barrido no detecta el óxido de cobalto y es esta inexistencia lo que permite saber que nos encontramos ante este pigmento. Pero en el caso tacorontero, aparece continuamente con picos, en algunos casos, de más del 2,5\%. Esto viene a confirmar la teoría de que el artesonado fue pintado para ser visto, admirado y con una función religiosa asociada a la imagen cristológica que preside el santuario.

Todo lo expuesto hace pensar que, tanto la policromía como el dorado no tienen una simple función decorativa, sino que existe una clara motivación animista en la que el pintor, a la manera de las grandes pinturas rupestres figurativas paleolíticas, pone todo aquello que enriquece y alimenta al municipio (las materias primas resultantes de su producción agrícola y ganadera, además de bienes, enseres y demás elementos de la vida cotidiana y comercial), en un intento por conseguir el beneplácito y la protección del Cristo Varón de Dolores, imagen expuesta en el retablo mayor y que fue devocionada a partir de 1662, tras un año de su llegada y de análisis por parte del Tribunal del Santo Oficio ${ }^{12}$. No es de extrañar que, transformada en una de las iconografías cristológicas más veneradas de las Islas Canarias, todos los tacoronteros se pusieran al servicio de su sagrada imagen, ofreciéndole todas sus pertenencias a través de las representaciones pictóricas en la cubierta. La riqueza de los materiales no hace sino atestiguar y fundamentar esta teoría del arte al servicio de la devoción y admiración de un pueblo.

12 Ruiz Álvarez, A. (1953). La Inquisición en Canarias y el Cristo de Tacoronte. Revista de Historia Canaria, (101-104), pp. 174-180. 
Microscopio electrónico de barrido y elucubraciones históricas. El artesonado mudéjar del santuario de Tacoronte (Tenerife)

\section{RECONOCIMIENTOS}

Gracias al Departamento de Conservación y Restauración de Bienes Culturales de la Universidad Politécnica de Valencia, con mención especial a la catedrática D ${ }^{a}$. Ma. Victoria Vivancos Ramón y a la Dra. Dª . Dolores Julia Yusá Marco, así como al Instituto de Restauración del Patrimonio, perteneciente a la misma institución.

\section{BIBLIOGRAFÍA}

Acosta, R. (2005). Las techumbres mudéjares policromadas en Canarias. Estudio de tres casos. En VIII Simposio sobre centros históricos y patrimonio cultural de Canarias. San Cristóbal de La Laguna, España: Fundación CICOP.

Bonnet, S. (1944). Tacoronte y sus templos. El Museo Canario, V(11).

Bonnet, S.F. (Noviembre 1961). El convento y la iglesia de San Agustín, en Tacoronte. La Tarde.

Calvo, A. (1997). Materiales, técnicas y procedimientos. De la A la Z. Barcelona, España:

Del Serbal.

Casas, J. (1987) Estudio Histórico Artístico de Tacoronte. Santa Cruz de Tenerife, España:

Aula de Cultura de Tenerife, Excmo. Cabildo Insular de Tenerife, 1987.

Cennino, C. (1957): El libro del arte. Buenos Aires, Argentina: Argos.

Fraga González, M. C. (1977) La arquitectura mudéjar en Canarias. Santa Cruz de Tenerife, España: Aula de Cultura de Tenerife.

González-Alonso Martínez, E, (1997). Tratado del dorado, plateado y su policromía.

Tecnología, conservación y restauración. Valencia: Servicio de Publicaciones de la Universidad Politécnica de Valencia.

Marrero, A. (2012) Artesonado de la Capilla Mayor del Santuario del Santísimo Cristo de Tacoronte: Desarrollo Iconográfico y su Interpretación. Revista de Historia Canaria (194). Marrero, A. (2013). Los Artesonados Mudéjares Policromados en la isla de Tenerife.

Aspectos técnicos y su conservación y restauración. En UPV (Ed.), Trabajos de Fin de 
Microscopio electrónico de barrido y elucubraciones históricas. El artesonado mudéjar del santuario de Tacoronte (Tenerife)

Máster: Máster Universitario en Conservación y restauración de Bienes Culturales 2006/2010. Trabajos seleccionados. Valencia, España: Universidad Politécnica de Valencia.

Marrero, A. (2016). Los Artesonados Mudéjares Policromados en la Isla de Tenerife (Tesis doctoral). Universidad de La Laguna, San Cristóbal de La Laguna.

Marrero, A. (2017) ¿Mudéjar o Mudejárico? Compendio historiográfico y reflexión crítica. El uso del término mudéjar en canarias. Revista de Historia Canaria (199).

Marrero A. (Julio - Diciembre 2018). Armaduras: planos póstumos y reconstrucción. La Iglesia de la Concepción (Los Realejos). Revista Estoa (13).

Mayer, R. (1993). Materiales y técnicas del arte. Madrid: Hermann Blume Ediciones.

Ruiz, A. (1953). La Inquisición en Canarias y el Cristo de Tacoronte. Revista de Historia Canaria, (101-104).

Vivancos, V. (2007). La conservación y restauración de pintura de caballete. Pintura sobre tabla. Madrid, España: Tecnos. 
Microscopio electrónico de barrido y elucubraciones históricas. El artesonado mudéjar del santuario de Tacoronte (Tenerife)

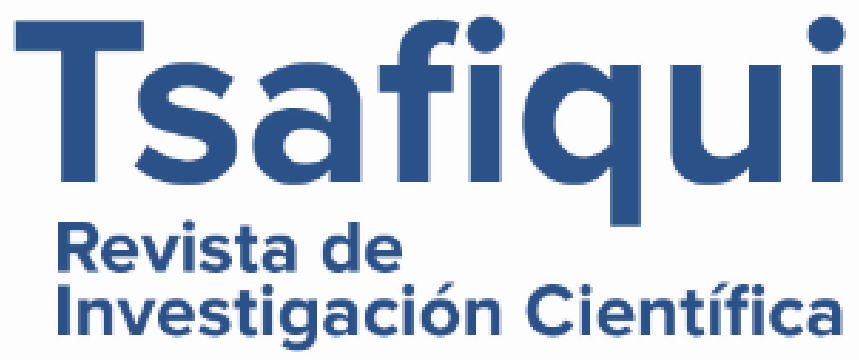

